\title{
Ataque mortal por perros de raza rottweiller a una mujer adulta: posible ataque en manada y antropofagia postmortal. Revisión y análisis de un caso de autopsia médico legal
}

\author{
Death of an adult woman after attack by two rottweiller \\ breed dogs: discussion about "pack attack" and postmorten \\ anthropophagy. A case report and literature review
}

\begin{abstract}
Resumen
Se presenta un infrecuente caso de muerte de una mujer adulta por posible ataque no presenciado de dos perros de raza rottweiller que se hallaban en su casa para guarda de la finca. La interpretación del caso indica, sin embargo, un posible ataque mortal por parte de uno solo de los dos animales con conducta posterior de antropofagia postmortal tras dar muerte a la mujer. Se revisa la literatura médicolegal reciente y se analizan los aspectos epidemiológicos de este tipo de muerte judiciales, incidiendo especialmente en los rasgos diferenciales de las lesiones producidas por los perros en casos de ataques mortales. Se reseña también la conveniencia de la realización de autopsia veterinaria y recogida de muestras para estudios de genética forense en estos casos, así como las ventajas y limitaciones del uso de los moldes dentales caninos para la reconstrucción de las lesiones.
\end{abstract}

Palabras clave: Lesiones. Muerte. Autopsia médico legal. Perro. Mordedura. Antropofagia. Rottweiller.

\section{Abstract}

We present a case report of an unsual unwitnessed violent death of an adult woman supposedly killed by her two guardian dogs. Both dogs were rottweiller breed. The conclusion of the medicolegal investigation indicates that the woman was probaly attacked by only one of the dogs and after death one or both dogs ate anatomic parts of the body (head). A review of updated medicolegal publications was made. The results of the review were centered on epidemiological profile of death by dogs attack, the features of mortal dog attacks and the features of the injuries produced by dogs on death bodies. This review confirms the unusual characteristics of this medicolegal investigation. Some recommendations like performing a veterinary autopsy in all cases and taking biological samples for forensic genetics investigations were included. Advantages and limitations of bite-injury analysis by odontological methods in complicated cases like the one reported in this paper were also discussed.

Key words: Injuries. Death. Medicolegal autopsy. Dog. Bite-injuries. Anthropofagy. Rottweiller.

\section{Introducción}

La incidencia exacta de muertes humanas provocadas por perros no es bien conocida. En EE.UU. se estima que cada año pueden morir por ataques de estos animales 73 personas por 100 millones de habitantes; entre 1979 y 1998 se registraron 238 muertes por tales ataques en este país ${ }^{1-4}$. En Japón esta cifra es de apenas unos pocos casos por año ${ }^{1}$. En España, las estadísticas también son incompletas ${ }^{5}$.
Así, entre 1991 y 2005 se informó en los medios de comunicación de 17 muertes por ataques de perros. Durante 2010 se informó en los medios de comunicación de al menos 5 muertes por estos ataques.

En EE.UU., considerada una población total estimada de perros, solo se relacionan con muertes humanas un 0,00000003\% de los perros. En España, con una población estimada de en torno a 4 millones de perros ${ }^{6}$ y estimando aproximadamente 5 muertes por perros durante el año 2009, la tasa de muertes
PM. Garamendi ${ }^{1}$

M. López Alcaraz ${ }^{2}$

${ }^{1}$ Médico Forense. Resident Twinning Adviser.

Twinning Project "Improving the skills of Forensic Experts (TR 08 IB JH 01)". Consejo de Medicina Forense de Estambul (Turquía).

${ }^{2}$ Médico Forense. Servicio de Patología Forense. IML de Huelva (España).

Correspondencia: Pedro Manuel Garamendi Adli Tıp Kurumu (Council of Forensic Medicine) Yenibosna Cobançeşme Mah.Sanayi Cad. Kımız Sok.No:1 Bahçelievler / İstanbul 34196

Email:imanolgaramendi @gmail.com

Fecha de recepción: 25.ENE.2012

Fecha de aceptación: 25.MAY.2012 
humanas por estos animales fue de 1,25 muertes por millón de perros para el año 2009; en el mismo año, el número de casos de homicidio consumado producidos por humanos en España fue de 337, con una tasa relativa a la población española estimada para ese año de 7,4 homicidios por millón de habitantes ${ }^{7}$. De ello se deduce que el número de muertes humanas provocadas por perros en nuestro país fue aproximadamente 5,90 veces menor que el número de casos de homicidios consumados y producidos por seres humanos.

Las muertes provocadas por perros, especialmente en aquellos casos de ataques no presenciados, conIlevan una serie de dificultades que complican la reconstrucción efectiva de los hechos. Diversas cuestiones surgen en el curso de la investigación de estos escenarios. ¿La muerte fue provocada por el ataque de un animal? En este caso, ¿fue un perro u otro animal? ¿Las lesiones apreciadas fueron provocadas antes o después de la muerte? ¿Cuál fue la secuencia de las lesiones? ¿El ataque fue provocado por uno o por varios perros y cuál de ellos pudo ser el responsable de las lesiones mortales? ¿Cuál fue la motivación del ataque? ¿Existió agresión sexual u otros tipos de violencia asociados? ¿Es la antropofagia una conducta canina habitual? El conocimiento de algunas características propias de las conductas agresivas de estos animales, de sus patrones lesivos y de las herramientas de investigación más adecuadas y sus limitaciones pueden colaborar junto con una investigación médico legal y en especial del lugar de los hechos minuciosas a la reconstrucción más certera de estos escenarios.

\section{Presentación del caso}

Se presenta un caso de autopsia médico legal de una mujer de 46 años de edad, 1,57 metros de estatura y biotipo normosómico delgado. Es hallada en el patio de su casa de campo en posición de decúbito supino, con las ropas removidas y empapadas en sangre en su parte declive (Figura 1). El patio se halla embaldosado, sin techo y con pendiente que se dirige hacia la puerta exterior del mismo que da a un campo. Las piernas, vestidas con medias y botas altas, se hallan cruzadas. Las ropas en la parte superior del cuerpo se hallan removidas. Consta asistencia sanitaria previa, pero no información exacta sobre las maniobras realizadas durante ésta. Bajo la víctima y a su alrededor se hallan restos escasos de sangre. La noche anterior al hallazgo de la víctima había llovido profusamente y las ropas y la superficie del patio se hallan húmedas. Junto al flanco derecho de la víctima se halla un mechón de cabellos negros cortos análogos a los del cabello de la víctima. En la entrada al lavadero del patio se hallan restos de sangre vomitada con algunos restos de cabellos análogos a los hallados junto a la víctima.

El examen de la casa y del resto de la hacienda no indica signos de violencia. En la entrada de la hacienda, con la puerta de esta abierta, se halla el coche de la víctima, con el motor en posición de encendido, las luces puestas y la puerta abierta. A unos metros de la víctima se halla una linterna con las baterías agotadas y en posición de "on".

Figura 1.

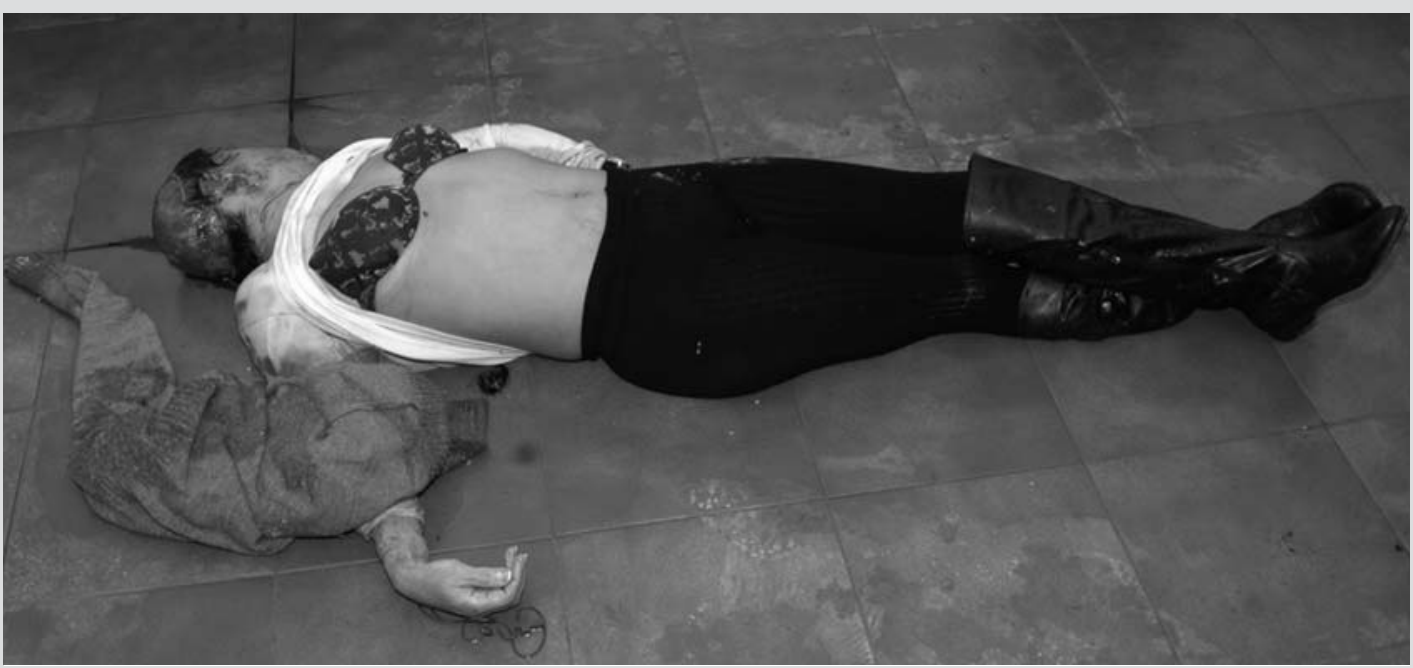


El cadáver presenta al menos seis de los siete patrones de lesión característicos descritos en la literatura médico legal en muertes por ataques de perros ${ }^{1-4,8-13}$. Las lesiones se hallaron localizadas en:

\section{Región craneal:}

- Ausencia de cuero cabelludo casi completa con exposición completa de plano galeal. Pérdida parcial de aponeurosis galeal. Bordes de sección corto-contusos (Figura 2).

- Amputación completa de pabellón auricular izquierdo y parcial del derecho. Heridas con bordes sangrantes irregulares.

- Múltiples heridas en ojal en ambos hemirrostros y labios.

\section{Extremidad superior derecha:}

- Heridas puntiformes punzo-contusas múltiples (Figura 3)

- Heridas amplias en ojal de eje mayor perpendicular al eje del miembro, de características punzo-cortantes y bordes contusos (Figura 4).

- Desgarro irregular completa de masas musculares. Destrucción de aponeurosis. Desinserción del plano dérmico y subdérmico con puentes de tejido fibroso que mantienen parcialmente la continuidad subdérmica (Figura 5).

\section{Región cervical:}

- Erosiones lineales (compatibles con lesión por garras) (Figura 6), así como lesiones punzo-cortantes contusas en ojal.

Tronco, extremidades inferiores o extremidad superior izquierda: sin lesiones.

Los hallazgos de la autopsia completa practicada a la fallecida únicamente evidenciaron la presencia de las lesiones ya descritas, de características vitales y/o perimortales y hallazgos compatibles con hemorragia importante y shock asociado, sin hallazgos indicativos de otras causas de muerte naturales o traumáticas. El estudio toxicológico fue negativo.

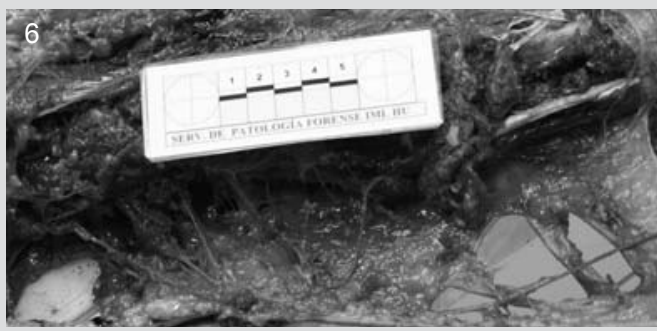

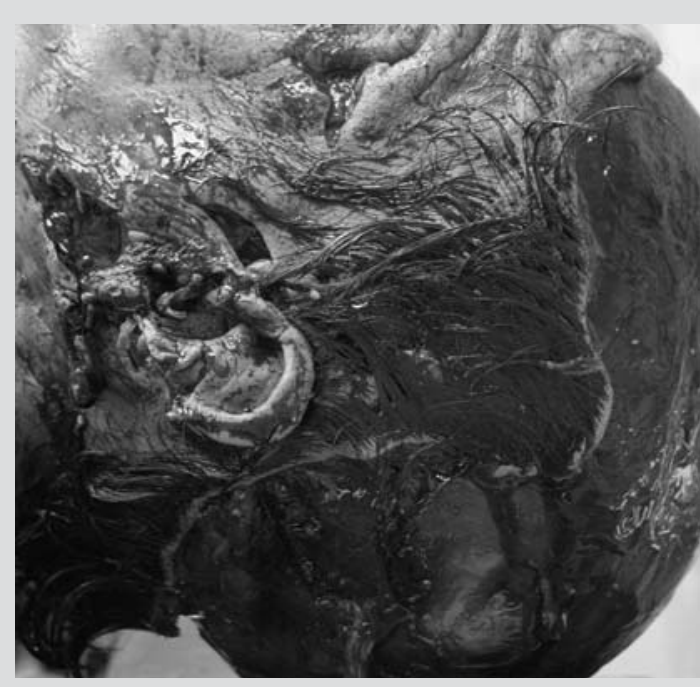

\section{Figura 2.}

Ausencia de cuero cabelludo. Lesiones con bordes cortantes y componente contuso.

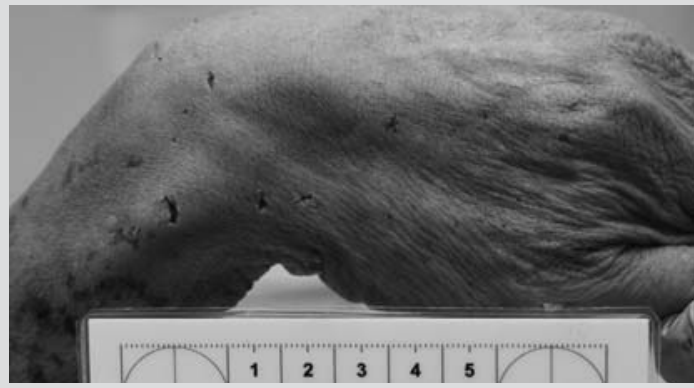

Figura 3.

Lesiones puntiformes en antebrazo derecho. Detalle de mano.

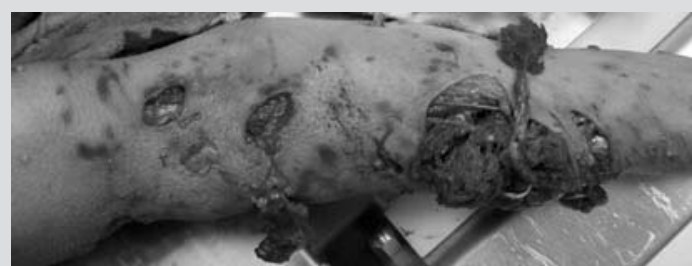

\section{Figura 4.}

Lesiones en brazo derecho.

esiones por caninos corto-punzantes con componente contuso. Salida de masas musculares seccionadas por orificios.

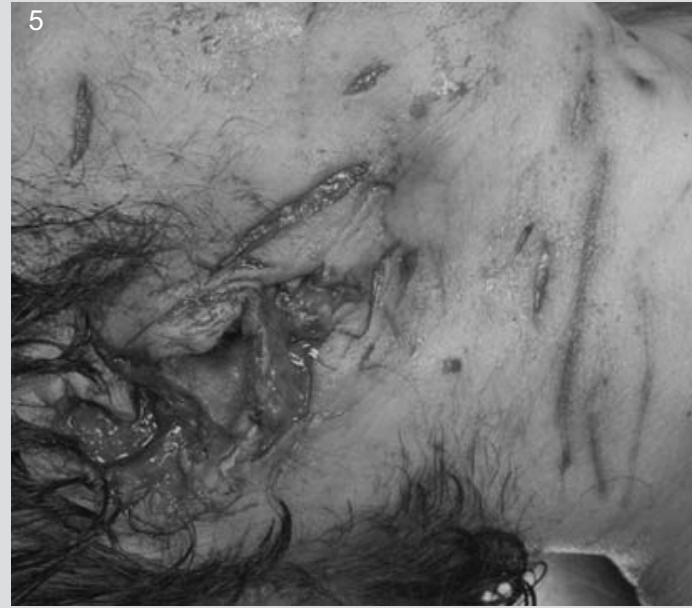

Figura 5

Erosiones lineales en cuello por garras.

\section{Figura 6.}

Desgarros de planos subcutáneos y puentes cutáneos en antebrazo derecho tras la disección cutánea. 
En la finca se hallan dos perros de guarda raza rottweiler, macho no castrado (1 año y 5 meses de edad) y hembra ( 9 meses de edad). Los perros no se hallaban atados y según parece el macho había mordido anteriormente a la mujer. No habían sido sometidos a educación canina o entrenamiento.

Tras la realización de la autopsia y con carácter diferido, la autoridad judicial recomienda el sacrificio de los animales. Se toman moldes dentales de los perros, sangre para estudio de ADN y muestras de las heces. En el perro macho, las heces presentan restos de cabellos negros de morfología levemente curvada de longitud y morfología compatibles con las del cabello de la fallecida. La autoridad judicial no ordena la autopsia veterinaria de los animales. Los estudios de genética forense solicitados y de análisis de cabellos en heces caninas no se llegan a completar en el laboratorio de referencia.

\section{Discusión}

Las víctimas mortales de los ataques por perros son miembros del grupo familiar al que pertenece el animal en un $70 \%$ de casos $^{8}$. En el 79,7\% de casos la agresión mortal sucede en la propiedad del dueño del animal ${ }^{2}$. En series de casos de lesiones no mortales, sin embargo, hasta en el $89,8 \%$ de los casos el perro responsable de la agresión es un animal ajeno al círculo familiar ${ }^{14}$.

Si bien las víctimas más frecuentes de este tipo de muertes suelen ser niños o personas incapacitadas, no es excepcional el ataque a adultos dueños de los perros, como se produjo en el caso que se presenta. Las víctimas más frecuentes son niños menores de 5 años, quienes en el curso del juego interpretan erróneamente las conductas amenazantes disuasorias previas de los animales $2,3,8$. No obstante, conductas de ataque y posterior antropofagia, como las apreciadas en este caso, no son habituales en caso de víctimas adultas, salvo en casos de antropofagia postmortal ${ }^{9}$.

Las razas más habitualmente asociadas en la literatura con este tipo de ataques suelen ser algunas de las etiquetadas como razas peligrosas como rottweiler, pit bull terrier o doberman. Menos comunes son los reportes de ataques mortales por razas también consideradas peligrosas como Akita o Tosa ${ }^{1}$ y por otras no consideradas peligrosas como pastor alemán o husky ${ }^{8}$. Curiosamente, en nuestro país una de las razas no incluidas en la lista del anexo I del RD 287/2002 de razas peligrosas, el pastor alemán, es responsable de la mayoría de ataques mortales reportados $^{5,6}$. La incidencia en cada país es muy distinta, posiblemente reflejo de la diversa proporción de perros de cada una de estas razas en cada país. Los ataques mortales se produjeron principalmente por parte de perros macho, de 6 a 12 meses de edad, no castrados y encadenados ${ }^{2,3}$. Los perros caIlejeros suelen provocar episodios de agresión menos graves, siendo la mayoría de casos mortales provocados por mascotas o perros de guarda ${ }^{2,3}$. Se han reportado casos también de ataques mortales incluso en el caso de perros tradicionalmente considerados no peligrosos y de pequeño tamaño, como el cocker spaniel o el duschshound miniatura ${ }^{15}$.

La conducta agresiva en los perros se relaciona con diversas circunstancias: dominancia (por ejemplo, quitar un objeto de la boca), territorialidad (entrar en el territorio "propiedad" del animal o de su grupo familiar), miedo (presencia inesperada de extraños), predatoria (aparición de personas en movimiento), maternal (intentos de acercarse a la camada), redirigida (intentos de separar a dos perros enzarzados en una pelea) u orgánica (dolor del animal, hidrocefalia y otras enfermedades). Algún autor ha sugerido que la presencia de perras en celo en el entorno puede aumentar la agresividad de los perros machos. Sin embargo, no se ha demostrado que éste sea un factor significativo en casos de episodios de ataque mortal por perros ${ }^{16}$. Salvo en el caso de razas como el husky, que no emite ladridos o el pit bull terrier que tiene una mímica limitada, los ataques suelen ser precedidos de conductas amenazantes disuasorias: gruñidos, retracción de los belfos, mirada fija, orejas y cola alzadas, postura quieta, etc. En raras ocasiones, tras un análisis minucioso del caso, puede estimarse que el ataque del perro, considerada su etología, resultó impredecible e inmotivado. La incapacidad para comprender estas señales previas suelen subyacer tras los ataques efectivos de los perros ${ }^{5,17-19}$.

Los ataques más peligrosos desde el punto de vista del riesgo vital se dan en casos de ataques en manada por parte de varios perros (pack attack) ${ }^{20}$. En estos casos, dos o más perros atacan a la víctima por varios frentes hasta derribarla y dejarla indefensa ${ }^{3}$. Por ello, es común hallar lesiones localizadas en diversas regiones corporales, con planos de ataque contrapuestos y con frecuentes lesiones en miembros inferiores destinadas a abatir a la víctima, hallazgos que no se hallaban presentes en el caso que se presenta.

Los lugares anatómicos más comúnmente lesionados son los miembros inferiores (en torno al $60 \%)^{8}$. Son menos comunes las lesiones en la cabeza y cuello, excepto en casos mortales ${ }^{9}$. Las lesiones en el plano cervical y facial habitualmente se relacionan más comúnmente con ataques a niños pequeños, en los que 
el plano facial queda a la altura de ataque de la cabeza del animal. Los ataques mortales se han descrito asociados en ocasiones exclusivamente en la región de la cabeza y cuello, achacándose la muerte en esos casos a un mecanismo combinado de shock neurogénico e hipovolémico por heridas en el cuero cabelludo. Se han descrito también otros mecanismos de muerte menos comunes asociados con lesiones en el plano cervical (arteria vertebral, médula cervical y obstrucción de vías aéreas) ${ }^{1,9}$.

En el caso que se presenta, en el que la víctima fue una mujer adulta, resulta excepcional el hallazgo de lesiones de características vitales localizadas en una sola extremidad superior y, sobre todo, en el cuero cabelludo y cara, con denudación de éste, ingestión del mismo y sin lesiones localizadas en la región cervical o en las extremidades inferiores.

La morfología característica de la lesiones está relacionada con la forma peculiar de la dentición canina, con sus hábitos de ataque y con el uso combinado de las garras de sus patas delanteras ${ }^{8}$. La dentadura de los perros tiene una funcionalidad diversa de la de los humanos. La dentadura definitiva de los perros consta de 42 piezas dentales. La arcada maxilar tiene una mayor amplitud que la mandibular, lo que permite el encaje de los incisivos "en tijera". Los incisivos (3 en cada hemiarcada), con característica morfología en flor de lis, tienen una función específica de separación de partes blandas. También son usados a modo de pinzas en las maniobras de autodesparasitado. Los caninos (1 en cada hemiarcada) tienen morfología cónica alargada curvada atrás y hacia fuera, siendo mayores los de la arcada maxilar (Figura 7). Su función es la de penetrar las partes blandas y así atrapar y sujetar a la pieza. Los premolares (4 en cada hemiarcada) tienen la función de cortar y desgarrar. Las caras cortantes de los premolares se oponen entre sí de modo que se superponen al cierre mandibular con un efecto cortante de sierra dentada. El cuarto premolar superior, mayor que los demás, se opone al primer molar inferior y entre ambos se produce un efecto de cizalla que permite desgarrar la carne en trozos, por lo que se denomina a ambos dientes carniceros (Figura 8). Los molares ( 2 en la hemiarcada superior y 3 en la inferior), de tamaño decreciente, tienen una cara externa cortante y una lingual molariforme, que utilizan habitualmente para triturar las piezas óseas y exponer el tuétano ${ }^{21}$.

Cuando se produce la conducta de ataque el perro atrapa y sujeta a su víctima con los caninos. Una vez sujeta por la presa canina, agita su cuerpo y su cabeza de modo que los caninos profundamente introducidos desgarran ampliamente los planos blandos profundos. Hallándose la víctima inerme utiliza las piezas premolares para cortar las partes blandas (dientes carniceros) y los incisivos para separarlas de las partes óseas. En caso de necesitar triturar hueso o cartílago utiliza las piezas molares. Para sujetar a la pieza y evitar que escape a la presión mandibular utiliza las patas delanteras para fijarla, presionando con sus garras sobre la superficie ${ }^{8}$.

La fuerza de presión de la mandíbula de los cánidos está influida por varios factores: tamaño del animal, tamaño de la cabeza, longitud de la mandíbula y entrenamiento. En términos generales, puede decirse que los perros de mayor tamaño, con mayor musculatura craneal, de mandíbula más corta y con entrenamiento previo tienen mayor fuerza de presión mandibular. Esta fuerza puede llegar a ser de hasta 300 kilogramos de fuerza en el caso de perros entrenados de razas grandes como el rottweiler, gran danés o el mastín, en oposición a otras razas como el galgo con menor fuerza de presión ${ }^{8}$.
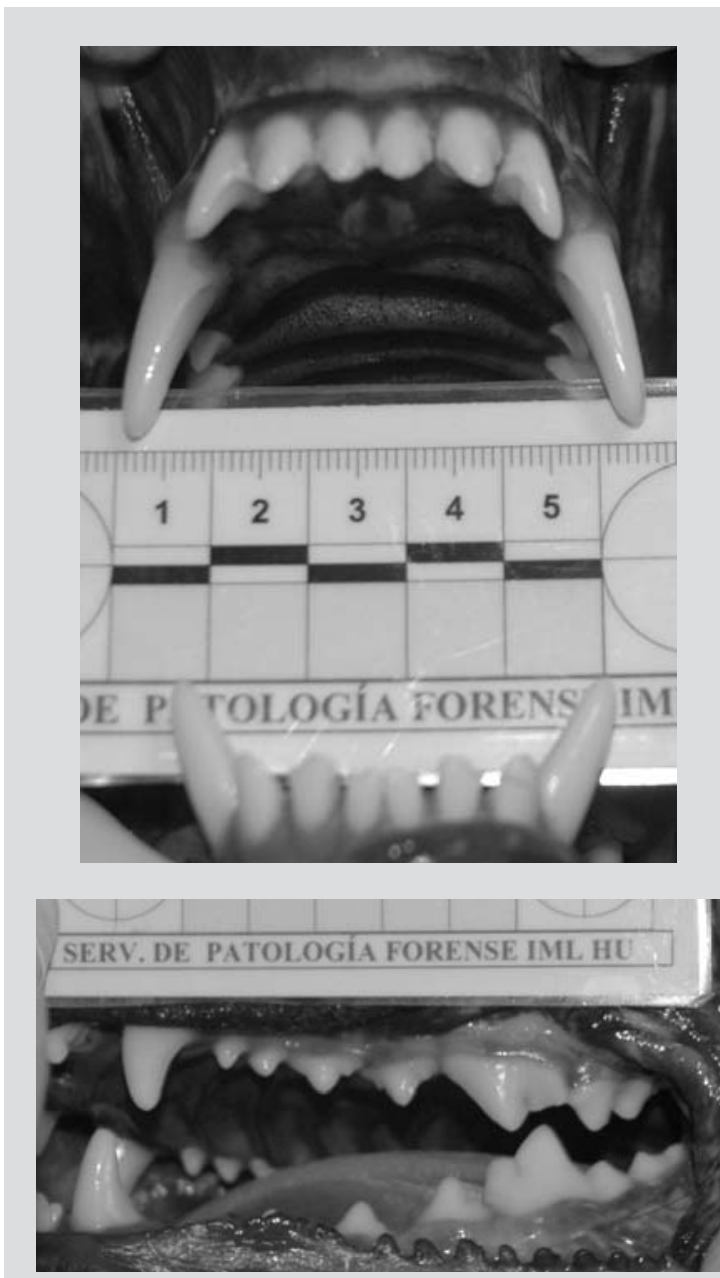

Figura 7.

Dentición de perro macho. Distancia entre caninos.

Figura 8.

Dientes carniceros. Perro hembra. 
De este modo, en casos de ataques mortales por perros, es posible encontrar varios tipos de lesiones habitualmente combinadas ${ }^{1-4,8-13}$ :

- Lesiones contuso-punzantes: producidas por la acción de los caninos cuando penetran en el plano cutáneo sin mecanismo de sacudida asociado.

- Lesiones inciso-punzantes con características contusas: producidas por penetración de los caninos en el plano cutáneo y desplazamiento al agitar la cabeza.

- Desgarros amplios de planos profundos: producidos habitualmente tras la presa canina al agitar el cuerpo el perro para desgarrar la presa.

- Amputaciones: por acción de molares o los incisivos en el curso del ataque o tras derribar a la víctima.

- Lesiones inciso-contusas: por acción de los molares al cortar la piel.

- Erosiones lineales: producidas por la acción de las garras de las patas delanteras. Se pueden producir tanto durante el ataque como, una vez derribada la víctima, al intentar evitar que los miembros se desplacen al cortarlos.

- Lesiones óseas: puntiformes por la acción de los caninos, surcos por la acción de los incisivos, fracturas producidas por los molares.

- Otras lesiones: ropas removidas, cuerpo colocado en posiciones inusuales (como las piernas cruzadas) y lesiones por arrastre en los planos de apoyo del cuerpo provocadas por el zarandeo de la víctima.

En el caso que reportamos, todos los patrones lesionales descritos se pudieron identificar, excepto las lesiones a nivel óseo. Estas lesiones óseas, habituales en otros casos, no fueron evidentes en el curso de la autopsia, si bien posiblemente podrían haber sido evidenciadas también en caso de haber realizado un

Figura 9. Moldes dentales del perro macho. Caninos superiores.

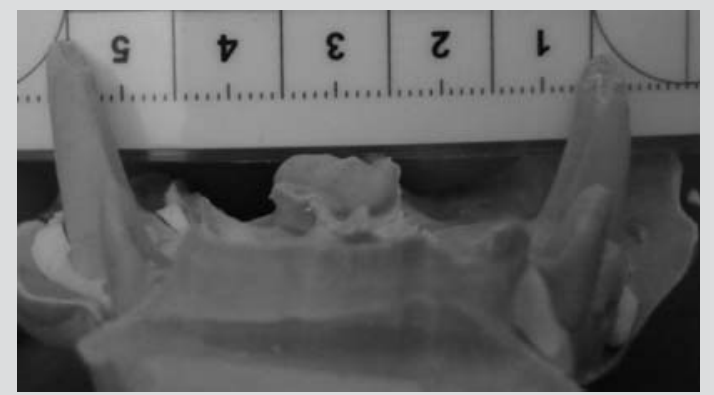

tratamiento antropológico de los restos óseos con el fin de exponer los planos corticales óseos y poder de ese modo valorar mejor las trazas en su superficie.

Es necesario resaltar que en la mayoría de los casos, las víctimas de ataques por perros suelen aparecer parcialmente desnudas, creando la sospecha de una posible agresión sexual ${ }^{8}$. Excepcionalmente también se ha podido apreciar la presencia de restos de esperma de los perros agresores en el cuerpo de la víctima sugiriendo un posible ataque sexual asociado ${ }^{22}$.

En el caso que ocupa este reporte, las ropas se hallaban removidas y las piernas en postura cruzada, pero sin evidencias de remoción a nivel de la región de pelvis y extremidades inferiores (Figura 1). La ropa pudo haber sido removida durante el ataque, en el que los perros suelen agitar y tironear del cuerpo de la víctima, haciendo posible el desnudamiento parcial de la víctima y su disposición en actitudes inhabituales. El estado intacto de las ropas en las extremidades inferiores y el examen genital negativo no sugirieron agresión sexual.

Las lesiones cutáneas pueden presentar una morfología característica útil para el odontólogo forense en la identificación de especie, raza y, se ha sugerido, del sujeto responsable del mordisco. Algunos autores destacan que la presencia de 6 incisivos en la mordida, con el arco frontal entre los caninos curvado, la configuración no oval de la lesión completa (como en mordeduras humanas) y la presencia de profundas marcas de caninos son sugestivas de mordeduras por mamíferos de la familia de los cánidos (lobo, coyote, perro). La distancia entre las marcas de los caninos es sugestiva de la distancia entre los caninos del animal y por ello puede usarse como indicador del tamaño del animal21,23,24. Algunos autores recomiendan la toma de moldes dentales para superposición de las heridas con dichos moldes y para la conservación de piezas de convicción fehacientes de los parámetros de la dentición animal, algunos como la distancia intercaninos con eventual utilidad reconstructiva en casos de heridas simples por mordedura canina (Figura 9). Se ha sugerido también que algunas características distintivas de la dentición del animal (rotaciones, malposiciones o ausencias de piezas dentales) pueden reflejarse en las marcas de mordedura. No obstante, algunos recientes trabajos alertan sobre el uso de estas marcas de mordedura con fines de identificación basada en características específicas de la dentición ${ }^{25,26}$. Así, en experimentos sobre mordeduras humanas se ha podido identificar la presencia de falsos positivos a hallazgos como diastema, rotaciones de piezas dentales o incluso la falsa imagen positiva de piezas dentales en realidad ausentes ${ }^{25,26}$. Las características del complejo 
comportamiento elástico de la piel, de respuesta impredecible a los efectos de presión, han llevado a sugerir el uso con extremada precaución con fines de identificación de las marcas de mordedura ${ }^{27,28}$. Especialmente en escenarios tan complejos como los manifestados por el caso que se presenta, con evidencia de múltiples lesiones por mordedura superpuestas y con muy diversos grados de presión y afectación de los planos cutáneos e incluso disrupción de la continuidad cutánea y de su anclaje a los planos subcutáneos, dichas estimaciones deberían ser extremadamente cautelosas. Por esta razón, aunque se obtuvieron moldes dentales de los perros en el caso que se presenta se decidió no hacer reconstrucción alguna de las lesiones basada en ellos.

En los casos de ataques postmortales por perros sobre cuerpos fallecidos, no es extraño que los animales se alimenten con restos del cuerpo del fallecido. Se ha interpretado esta acción en el contexto de situaciones de hambre de mascotas que permanecen largo tiempo junto a sus dueños fallecidos, pero también se ha asociado con situaciones en las que el hambre se ha descartado como factor asociado explicándose por estados de ansiedad de las mascotas que intentan infructuosamente reanimar a sus dueños muertos. La conducta antropofágica no es habitual en casos de ataques vitales, si bien tampoco resulta absolutamente excepcional que los perros atacantes lleguen a devorar los restos del fallecido, especialmente en casos de ataques a menores 9,16,29,30,31.

Se suele recomendar la toma de muestras para estudio biológico de la superficie del cadáver, sangre del animal y de su contenido intestinal y saliva para buscar evidencias de mordedura y/o deglución de partes anatómicas de la víctima. Sin embargo, las técnicas de identificación genética informan únicamente sobre el contacto del animal identificado con la víctima. En este sentido es preciso indicar que no es extraño que las mascotas laman la superficie del cuerpo de sus dueños fallecidos en intentos de reanimación o para alimentarse de la sangre del fallecido, por lo que la presencia de vestigios genéticos positivos del animal en el cuerpo de la víctima no son absolutamente indicativos de la causación de la muerte por parte del animal. Del mismo modo, la identificación de restos genéticos del fallecido en restos hallados en el aparato digestivo del animal durante la autopsia sólo confirma la deglución de restos del fallecido por parte del animal que pudo producirse postmortalmente ${ }^{16,32-34}$. En el caso que se presenta, se solicitaron estos estudios, pero el laboratorio de referencia para análisis genéticos decidió unilateralmente no realizarlos.

La evaluación integral de los hallazgos de autopsia y el patrón lesional así como del lugar de los hechos en el caso que se presenta, pese a que en una primera aproximación sugirió la posible existencia de un ataque en manada ("pack attack") por los dos perros presentes en la hacienda, orientó sin embargo hacia un interpretación final en la que sólo uno de ambos perros posiblemente pudo ser responsable de todas las lesiones en la víctima. Las lesiones en el brazo derecho pudieron deberse al ataque inicial por parte de uno de los perros, con posible forcejeo, zarandeo de la víctima durante la presa y caída al suelo, dada la diferencia de fuerza y peso entre perro y víctima. Una vez en el suelo el animal pudo atacar el polo cefálico y con la víctima inconsciente por el shock neurogénico e hipovolémico o muerta pudo arrancar el cuero cabelludo con su dentadura y deglutirlo. Durante el acto de ataque al cuero cabelludo posiblemente usó las patas delanteras para fijar por el cuello la cabeza y poder más fácilmente realizar el arrancamiento. Esta modalidad de ataque con lesiones en la cabeza y deglución del cuero cabelludo perimortal en una víctima adulta resulta excepcional en la literatura médico legal consultada1-4,8-12,16,20,30,31,35.

El estudio reconstructivo de las lesiones mediante técnicas odontológicas no fue eficaz para poder distinguir entre lesiones producidas por uno u otro animal, dada la gran cantidad de lesiones existentes, su solapamiento y el alto grado de distorsión motivado por la destrucción de los planos de anclaje cutáneo. Posiblemente, en un escenario lesional menos complejo, estas técnicas de reconstrucción de mordeduras podrían haber tenido una mayor utilidad. EI estudio antropológico de los huesos podría haber colaborado a evidenciar lesiones corticales no evidentes en el estudio macroscópico, pero no llegó a realizarse. El estudio genético forense, que no llegó a completarse por parte del laboratorio receptor, podría haber colaborado a explicar en más detalle las condiciones de la agresión y de la posible deglución de los restos humanos por parte de uno o ambos perros.

En la Tabla 1 se resumen los datos más significativos recomendados por los distintos autores citados en la investigación de los casos de muerte por ataque de perros.

Las investigaciones de escenarios de muerte tras ataque por perros deben ser evaluadas de forma cuidadosa. El seguimiento minucioso de las pautas recomendadas por autores previos y la realización de un examen cuidadoso de la escena de los hechos, de los animales sospechosos, así como la entrevista a testigos puede evitar cometer graves errores de interpretación. Este tipo de escenarios resultan especialmente complejos y en ellos no debe descartarse la posible interferencia de otros tipos de violencia asociada que pueden quedar enmascarados en una primera aproximación por una investigación incompleta de los hechos ${ }^{35}$. 
Tabla 1.

Resumen de datos en investigación de la escena en caso de ataque mortal por perros.

\section{Examen de la escena}

- Datos generales:

Descripción de las circunstancias (descripción del ataque y de las situaciones previas al hecho).

Identificación de testigos.

Victima (edad, sexo, estado de salud, relación con el animal, interacción previa con el animal).

Animal (raza, sexo, edad, castrado o no, encadenado o suelto, comportamiento agresivo previo del animal).

Maniobras de resucitación (descripción completa).

- Examen de la escena:

Recogida de evidencias (manchas de sangre, etc.).

Condiciones ambientales,

Posición del cuerpo,

Posible lugar del ataque y desplazamientos.

- Documentación:

Fotografias y diagramas de la escena.

\section{Examen del cadáver}

-Datos generales:

- Edad, sexo, peso y talla,

Relación con el perro,

Estado de salud,

Perfil psicológico.

Examen externo (preferible en la escena de los hechos):

Marcas de mordedura (orificios y desgarros),

Heridas de defensa,

Lesiones y cambios postmortem,

Heridas y lesiones vitales y perimortales,

Estado de salud,

Recogida de evidencias,

Registro fotográfico y esquemas de lesiones (recomendable escala métrica ABFO 2).

- Examen interno:

- Recogida de muestras para estudio genético y toxicológico,

- Causa y mecanismo de muerte,

Descripción de lesiones mortales y no mortales.

- Toxicología: alcohol, drogas de abuso, etc.

- Análisis genético:

Identificación de cabellos del perro hallados sobre el cuerpo

Tomas de superficie corporal para estudio de restos de saliva del animal.

\section{Examen del animal}

- Contar con la colaboración, siempre que sea posible, de un patólogo veterinario.

- Datos generales:

Edad, sexo, raza, talla y peso,

Dueño,

Comportamiento previo

Historia sanitaria,

Relación con la víctima,

Datos de identificación (collares, tatuajes, chip canino).

- Examen externo:

Recogida de trazas (sangre, fibras de ropas, tierra, cabellos),

Estado de salud,

Heridas recientes,

Cicatrices (sugestivas de peleas previas o maltrato animal).

- Examen interno:

Autopsia completa,

Examen minucioso de la cavidad oral

Recogida de moldes dentarios y/o fotografías de la dentición,

Hallazgos patológicos,

Recogida de muestras de contenido de la boca, contenido gástrico, sangre, orina, tejido cerebral, etc.

- Toxicología: tóxicos, sustancias estimulantes.

- Microbiología: descartar rabia, infecciones. 


\section{Agradecimientos}

Miguel Zapata (Laboratorio de Criminalística de la Guardia Civil de Huelva), Da . Fátima Minchón y D. José Rodríguez (IML de Huelva), por su impecable estudio de la escena de los hechos y colaboración en la práctica de la autopsia judicial.
Dr. Guillermo Barba, médico estomatólogo de Huelva, por su inestimable ayuda en el proceso de recogida de los moldes dentales.

Agustín Mazón, entrañable compañero del IML y amigo, que nos ayudó a reflexionar y a considerar con la correcta perspectiva éste como tantos otros casos del servicio de patología forense del IML de Huelva.

\section{Bibliografía}

1. Oshima T, Mimasaka S, Yonemitsu K, Kita K, Tsunenari S. Vertebral artery injury due to fatal dog bites. J Forensic Leg Med. 2008;15:529-32.

2. Shields LB, Bernstein ML, Hunsaker JC, Stewart DM. Dog bite related deaths. A 15 year review of Kentucky Medical Examiner cases. Am J Forensic Med Pathol. 2009;30(3):223-30.

3. Loewe Ch, Diaz FJ, Bechinski J. Pitbull mauling deaths in Detroit. Am J Forensic Med Pathol. 2007;28(4):356-61.

4. Langley R. Human fatalities resulting from dog attacks in the United States, 1979-2005. Wilderness and Environmental Medicine. 2009;20:19-25.

5. Palacio J, León M, García-Belenguer S. Aspectos Epidemiológicos de las mordeduras caninas. Gac Sanit. 2005;19(1):50-8.

6. El Mundo-La Revista. Salvad a los perros españoles. Disponible en: http://www.elmundo.es/ magazine/ num187/textos/perros1.html (acceso 19-12-2011).

7. Web del Instituto Nacional de Estadística. Disponible en: http://www.ine.es (acceso 19-12-2011).

8. De Munnynck K, Van de Voorde W. Forensic approach of fatal dogs attack: a case report and review of the literature. Int J Leg Med. 2002;116:295-300.

9. Tsokos M, Byard R, Püschel K. Extensive and mutilating craniofacial trauma involving defleshing and decapitation: unusual features of fatal dog attacks in the young. Am J Forensic Med Pathol. 2007;28(2):131-7.

10. Calkins CM. Life threatening dog attacks: a devastating combination of penetrationg and blunt injuries. J Pediatr Surg. 2001;36:1115-7.

11. Tin D. Dog bite wounds in a child. Hong Kong Med J. 2007;13(3):247-8.

12. Jones NP. Perforating eyes injuries caused by dog bites. J Royal Soc Med. 1990;83:332-3.

13. Fischer H, Hammel PW, Dragovic LJ. Human bites versus dog bites. N Eng J Med. 2003;349:11.
14. Zannini F, Padinger P, Elissondo MC, Pérez H. Epidemiología de las lesiones por mordedura de perro en Tierra del Fuego, Argentina. Medicina (Buenos Aires). 2008;68:1-5.

15. Tsuji A, Ishiko A, Kimura $\mathrm{H}$ et al. Unusual death of a baby: a dog attack and confirmation using human and canine STRs. Int J leg Med. 2008;12259-62.

16. Borchelt PL, Lockwood R, Beck AM, Voith VL. Attacks by pack of dogs involving predation on human beings. Pub Health Reports. 1983;98(1):57-68.

17. Fatjo J, Ruiz de la Torre JL, Manteca X. The epidemiology of behavioural problems in dogs and cats: a survey of veterinary practitioners. Animal Welfare. 2006;15:179-85.

18. Bennett PC, Rohlf VI. Owner-companion dog interactions: Relationships between demographic variables, potentially problematic behaviours, training engagement and shared activities. Applied Animal Behaviour Science. 2007;102:65-84.

19. Beaver BV. Owner complaints about canine behavior. J Am Vet Med Assoc. 1994 Jun 15;204(12):1953-5.

20. Kneafsey B, Condon KC. Severe dog bite-injuries, introducing the concept of pack attack: a literature review and seven case reports. Injury 1995;26(1):37-4.

21. Murmann DC, Brumit PC, Schrader BA, Senn DR. A comparison of animal jaws and bite mark patterns. J Forensic Sci. 2006;51(4):846-60.

22. Wiegand P, Schmidt V, Kleiber M. German sheperd is suspected of sexually abusing a child. Int J Leg Med. 1998;112:324-5.

23. Pomara C, D'Errico S, Jarussi V, Turillazzi E, Fineschi V. Cave Canem: Bite marks analysis in a fatal dog pack attack. Am J Forensic Med Pathol. 2010 (ahead editing).

24. Ferrant O, Papin F, Dupont C Jr, Clin B, Babin E. Injuries inflicted by a pet ferret on a child: morphological aspects and comparison with other mammalian pet bite marks. J Clin Forensic Leg Med. 2008;15:193-7. 
25. Bowers CM, Pretty IA. Expert disagreement in bitemark casework. J Forensic Sci. 2009;54(4):915-8.

26. Bush MA, Cooper HI, Dorion RBJ. Inquiry into the Scientific basis for bitemarks profiling and arbitrary distortion compensation. J Forensic Sci. 2010;55 (4):976-84.

27. Bush MA, Miller RG, Bush PJ, Dorion RBJ. Biomechanical Factors in Human Dermal Bitemarks in a Cadaver Model . J Forensic Sci. 2009;54(1)167-76.

28. Bush MA, Thorsrud K, Miller RG, Dorion RBJ, Bush PJ. The response of skin to applied stress. investigation of bitemark distortion in a cadaver model. J Forensic Sci. 2010;55(1):71-6.

29. Verzeletti A, Cortellini V, Vassalini M. Postmortem injuries by a dog: a case report. J Forensic Leg Med. 2010;17:216-9.

30. Rotschild MA, Schneider V. On the temporal onset of postmortem animal scavenging: Motivation"of the animal. Forensic Sci Int. 1997;89:57-64.
31. Wright JC. Severe attacks by dogs: characteristics of the dogs, the victims and the attack settings. Pub Health Reports. 1985:100(1):55-61.

32. Budowle B, Garofano P, Hellman A, Ketchun M, et al. Recommendations for animal DNA forensic and identity testing. Int J Leg Med. 2005;119:295-302.

33. Tsuji A, Ishiko A, Kimura H, Nurimoto M, Kudo K, Ikeda N. Unusual death of a baby: a dog attack and confirmation using human and canine STRs. Int J Leg Med. 2008;122:59-62.

34. Eichmann C, Berger B, Reinhold M, Lutz M, Parson W. Canine-specific STR typing of saliva traces on dog bite wounds. Int J Leg Med. 2004;118:337-42.

35. Boglioli LR, Taff ML, Turkel SJ, Taylor JV, Peterson C, Denis DVM. Unusual Infant Death: Dog Attack or Postmortem Mutilation After Child Abuse? Am J Forensic Med Pathol. 2000;21(4):389-94. 